[0212-7199 (2006) 23: 1; pp 19-25] ANALES DE MEDICINA INTERNA Copyright (C) 2006 ARAN EDICIONES, S.L.

AN. MED. InTERnA (Madrid) Vol. 23, N. ${ }^{\circ} 1$, pp. 19-25, 2006

\section{Relación de factores clínico-bioquímicos con el estadio clínico y tipo de cirugía en una muestra de pacientes con isquemia crítica de miembros inferiores}

\author{
D. A. DE LUIS, H. OVALLE, J. C. VAQUERO, E. ROMERO
}

Instituto de Endocrinología y Nutrición. Facultad de Medicina. www.ienva.org. Valladolid

\section{RESUMEN}

Objetivo: El término isquemia crítica de miembros inferiores se utiliza en aquellos pacientes con isquemia crónica y dolor en reposo, úlcera o gangrena atribuible a la demostración objetiva de la enfermedad arterial oclusiva. Con el presente estudio observacional se pretende identificar los factores relacionados con el estadío clínico y el tipo de cirugía reparadora empleada en una población intervenida por arterioslerosis severa de miembros inferiores.

Material y métodos: Se ha realizado un estudio observacional durante un período de dos años, comprendido entre enero de 1999 y diciembre del 2000, con 330 pacientes intervenidos por enfermedad aterosclerótica severa de miembros inferiores, con isquemia crítica. A todos los pacientes se les recogieron, en el momento del ingreso para la cirugía, los siguientes datos: edad, sexo, tensión arterial, glucemia, colesterol total, LDL colesterol, HDL colesterol triglicéridos, topografia de la lesión, tipo de tratamiento quirúrgico, índice tobillo brazo, así como el tratamiento farmacológico prescrito para los factores de riesgo cardiovascular. Se realizó un analisis de regresión logistica para evaluar la influencia de las diferentes variables en la presencia de un estadio clinico IV frente al un estadío III. Así como, la utilización de amputación como técnica quirúrgica frente a revascularización.

Resultados: La edad media fue de 74, $78 \pm 10,35$ años, siendo la edad de las mujeres mayor $(78,88 \pm 10,45$ años) que la de los hombres $(73,70 \pm 10,07)(\mathrm{p}<0,0001)$, con una distribución por sexos, de 261 hombres $(79,1 \%)$ y 69 mujeres $(20,9 \%)$. El modelo de regresión logística construido para explicar el estadio clínico dicotomizado en III/IV, ajustado para la edad y sexo, las variables que mayor influencia tienen en el riesgo de padecer un estadio IV frente al III son la diabetes mellitus, la localización de la lesión arterial, y las cifras bajas de colesterol total. Un paciente con diabetes mellitus tipo 2 tiene 3,322 (IC 95\%: 1,881-5,866) veces más riesgo de presentar al ingreso un estadio clínico IV que una persona que no lo es. En cuanto al colesterol total, la OR es de 0,990 lo cual puede interpretarse como que el riesgo de presentar un estadio clínico IV frente al estadio clínico III se multiplicaría por 0,990 por cada $\mathrm{mg} / \mathrm{dl}$ de aumento del nivel de colesterol total. La localización de la lesión arterial distal (por debajo del ligamento inguinal) incrementa el riesgo de presentar un estadio clínico IV en 6,897 (IC 95\%: 3,509 13,557) veces más que la localización de la lesión arterial en otro sector.

El modelo de regresión logística construido para explicar el tipo de cirugía dicotomizado en amputaciones/derivaciones ajustado para la edad y sexo, la presencia de diabetes mellitus tipo 2 presenta una Odds Ratio (OR) de 3,37 lo que significa que el padecimiento de esta enfermedad multiplica por 3,37 (IC 95\%: 1,940-5,866) el riesgo de ser sometido a una amputación frente a una revascularización. Con respecto del colesterol total, la OR es de 0,986 lo cual puede interpretarse como que el riesgo de amputación frente a intervenciones de revascularización se multiplicaría por 0,986 por cada $\mathrm{mg} / \mathrm{dl}$ de aumento del nivel de colesterol
RELATION OF CLINICAL AND BIOCHEMICAL FACTORS WITH CLINICAL STAGE AND TYPE OF SURGERY IN A SAMPLE OF PATIENTS WITH CRITICAL ISCHEMIA OF LIMBS

\section{ABSTRACT}

Objective: The term of critical ischemia of legs is used in patients with chronical ischemia and pain in rest, ulcer or tisular necrosis secondary to oclussive arterial disease. The objective of our work was to identify factors related with clinical stage and type of surgery in these patients.

Material and methods: An observational study was performed during two years, from january 1999 to december 2000, with 330 patients with surgery secondary to critical ischemia of legs. The next parameters were recorded during the Hospital stance; age, sex, blood pressure, glycaemia, total colesterol, LDL colesterol, HDL colesterol, triglycerides, location of disease, type of surgery, arm-ankle index, and farmacological treatment to cardiovscular risk factors. A regression logistic analyze was realized to study the influence of diferents variables in the clinical stage and in the type of surgery.

Results: The mean age was $74.78 \pm 10.35$ years, with a higher age in females $(78.88 \pm 10.45$ years $)$ than males $(73.70 \pm 10.07)(p<0,0001)$, with 261 males (79.1\%) and 69 females (20.9\%). Logistic regression model to investigate clinical stage III/IV (adjusted by age and sex) showed that the independent variables related with stage IV were diabetes mellitus, location of arterial lesion, and low colesterol levels. A patient with diabetes mellitus type 2 has 3,322 (CI 95\%: 1,881-5,866) times more risk of stage IV than a non diabetic patient. Odds ratio of total colesterol was 0,990, the risk to develop a IV stage is increased 0,990 times for each $\mathrm{mg} / \mathrm{dl}$ of cholesterol. Distal lesion increased the risk of stage IV en 6,897 times (CI 95\%: 3,509 - 13,557).

Logistic regression model to investigate type of surgery (amputation/bypass) (adjusted by age and sex) showed that diabetes mellitus type 2 had Odds Ratio (OR) of 3,37, this means that diabetes increase 3,37 times (CI 95\%: 1,940-5,866) the risk of amputation. Total cholesterol showed a OR of 0,986, this jeans an increase of amputation 0,986 time with each $\mathrm{mg} / \mathrm{dl}$ of cholesterol. Distal location increase risk of amputation 2,585 times. Patients with more than one surgery had an OR of $3,013(1,602-5,666)$ to increase amputation. Each day of hospital stance increase $0,8 \%$ risk of amputation.

Conclusion: In multivariant análisis, diabetes mellitus, distal lesion, male sex and age over 75 years were positive related with stage $I V$, and colesterol levels were inverse related. Diabetes mellitus, distal lesion, age over 75 years, days of hospital stance, number of surgeries were positive related with amputation as surgery technic and colesterol levels were negative related. 
total. En cuanto al sitio de la lesión aunque la variable entra globalmente en el modelo, las diferencias significativas se sitúan en la comparación entre lesión distal y proximal siendo la OR de la primera de 2,585 en relación a la segunda. Las personas que han sufrido más de una intervención quirúrgica presentan una OR de 3,013 $(1,602-5,666)$ para sufrir una amputación frente a aquellas que han sido intervenidas una sola vez. Cada día de ingreso hospitalario aumenta en un $0,8 \%$ el riesgo de amputación frente a intervenciones de revascularización.

Conclusión: En el análisis multivariante la presencia de diabetes mellitus, lesiones distales, sexo femenino y edad mayor de 75 se relaciona con la presencia de un estadio IV, presentando los niveles de colesterol una relación inversa. La presencia de diabetes mellitus, lesion distal, más de una cirugía reparadora, edad mayor de 75 años, los días de ingreso se relacionan con al realización de amputaciones como técnica reparadora, presentando los niveles de colesterol una relación inversa.

PALABRAS CLAVE: Arteriosclerosis severa de miembros inferiores. Isquemia crítica de miembros inferiores. Factores clínicos. Tipo de cirugía.
KEY WORDS: Acute atherosclerosis in lower limbs. Acute ischemia of lower limbs. Clinical factors. Type of surgery.

De Luis DA, Ovalle H, Vaquero JC, Romero E. Relación de factores clínico-bioquímicos con el estadio clínico y tipo de cirugía en una muestra de pacientes con isquemia crítica de miembros inferiores. An Med Interna (Madrid) 2006; 23: 19-25.

\section{INTRODUCCIÓN}

El término isquemia crítica de miembros inferiores se utiliza en aquellos pacientes con isquemia crónica y dolor en reposo, úlcera o gangrena atribuible a la demostración objetiva de la enfermedad arterial oclusiva. Por tanto, la isquemia crítica crónica constituye una indicación absoluta de reconstrucción quirúrgica o amputación, si la reconstrucción quirúrgica no es posible (1).

Según algunas series, el 5\% de los pacientes con enfermedad arterial periférica (2) terminan con isquemia crítica de miembros inferiores. La mortalidad de la enfermedad arterial periférica, está estrechamente unida al estadiaje clínico, de este modo la presencia de dolor en reposo o pérdida tisular (isquemia crítica de miembros inferiores) empeora el pronóstico, con una tasa de mortalidad al año cerca del $20 \%$ (3).

Los factores de riesgo más importantes para la arteriopatía periférica y en la isquemia crítica de miembros inferiores, son la edad superior a 40 años, el consumo de tabaco y la diabetes mellitus, aunque la dislipemia, la hipertensión arterial también contribuyen a dicho riesgo $(1,4-5)$.

A pesar de los estudios existentes sobre factores de riesgo cardiovascular y supervivencia de estos pacientes, son escasos los trabajos que evaluen la relación existente entre estos factores y el estadío clínico o el tipo de cirugía empleada $(6,7)$.

Con el presente estudio observacional se pretende identificar los factores relacionados con el estadío clínico y el tipo de cirugía reparadora empleada en una población intervenida por arterioslerosis severa de miembros inferiores.

\section{PACIENTES Y MÉTODOS}

\section{PACIENTES}

Se ha realizado un estudio observacional durante un período de dos años, comprendido entre enero de 1999 y diciembre del 2000, en el cual se revisaron las historias clínicas en el momento del ingreso para cirugía de todos los pacientes intervenidos por arteriopatía crónica periférica, de causa aterosclerótica, en nuestro centro (330 pacientes).

\section{DIAGNÓSTICO DE ARTERIOPATÍA Y TIPO DE TRATAMIENTO}

Se recogieron los datos de localización de la lesión en el árbol arterial según los resultados de la arteriografía. Posteriormente esta variable fue dicotomizada en; lesión proximal (obstrucción de localización aorto-iliaca) y lesión distal (lesiones arteriales por debajo del ligamento inguinal, a partir de la arteria femoral superficial) (8). Se recogió el número de intervenciones quirúrgicas reparadoras realizadas en ese paciente.

Se recogieron los datos referentes al tipo de intervención quirúrgica realizada y posteriormente se dicotomizó en intervenciones de revascularización (aquellas intervenciones con técnicas de revascularización: bypass con injerto o autólogos, tromboendarterectomía, angioplastia transluminal percutánea, simpatectomía lumbar, etc.) y amputaciones (amputaciones mayores y menores del miembro inferior).

\section{SÍNTOMAS Y SIGNOS DEL PACIENTE}

Se recogieron los síntomas y signos del paciente en el momento del ingreso y expresamos los dos que clasifican el grado o estadio clínico de arteriopatía crónica periférica en el que se encontraban los pacientes; dolor en reposo y lesiones tróficas y/o gangrena. Según presentaran dolor en reposo o lesiones tróficas, clasificamos a los pacientes en estadio clínico III y IV respectivamente, según la clasificación de Fontaine (9) 


\section{INDICE TOBILLO/BRAZO (ITB)}

A todos los pacientes se les midió el ITB, presión arterial sistólica tomada en el tobillo/presión arterial sistólica en el brazo. Se consideraron patológicos los valores del ITB inferiores a 0,9 y muy patológicos los inferiores a 0,6. En los pacientes diabéticos, cuando el ITB fue mayor o igual a 1,25, ese valor se consideró sugestivo de calcificación arterial. Se utilizó un equipo doppler unidireccional portátil; Super Doppler- II modelo SD2, con transductor a $5 \mathrm{MHz}$. Con el transductor se buscó el punto en el que se oía mejor el latido arterial y se infló el manguito unos $20-30 \mathrm{mmHg}$ por encima de la desaparición del latido arterial; posteriormente se deshinchaba lentamente hasta la reaparición de dicho latido, momento considerado como el correspondiente al valor de la presión sistólica.

\section{FACTORES DE RIESGO CARDIOVASCULAR}

Recogimos el hábito tabáquico de los pacientes y la cantidad de cigarrillos que fumaban al día. Esta variable se dividió en fumadores (aquellos que fumaban al menos 1 cigarrillo diario, y a su vez agrupamos a aquellos que fumaban menos de 10 cigarrillos al día, los que fumaban entre 10 y 20 cigarrillos/día o fumadores moderados y los que fumaban más de 20 cigarrillos/día o grandes fumadores), exfumadores (los que habían abandonado el hábito de fumar al menos 6 meses antes de la recogida de datos) y no fumadores.

El consumo de alcohol permitió agrupar a los pacientes en no bebedores, exbebedores (los que habían abandonado el consumo diario de alcohol, 6 meses antes del comienzo del estudio) y bebedores (aquellos que consumían alcohol diariamente).

El peso corporal se midió mediante una balance con una precicion de $100 \mathrm{~g}$ y el IMC (indice de masa corporal) se calculó mediante la fórmula peso/(altura²). Para determinar el peso se utilizó una báscula automática digital, fuerza máxima de $200 \mathrm{~kg}$ (Atlántida. Barcelona). El tallímetro utilizado fue de marca (Atlántida. Barcelona) de 55-200 cm.

La presión arterial se midió dos veces con un esfingomanómetro de mercurio OMRON Mx3 (Omron Matsusaka Co. Ld, Japan), y se promediaron los resultados. Se consideró como hipertensión arterial (HTA) cuando las cifras de PAS $\mathrm{y} / \mathrm{o} \mathrm{PAD} \geq 140 / 90$ en tres determinaciones distintas.

Los niveles séricos de colesterol y triglicéridos se determinaron mediante ensayo enzimocolorimétrico (Automatic Analyzer, Amodelo 902, Tokio, Japan). Los niveles de HDL colesterol se determinaron mediante técnica enzimática en el sobrenadante, tras precipitación de las otras lipoproteínas con sulfato de magnesio. Los niveles de LDL colesterol se determinaron mediante la fórmula de Friedewald. Los niveles de glucosa se determinaron mediante técnica de glucosa oxidasa (Glucose analyser 2, Beckman Instruments, Fullerton, California).

Hemos considerado dislipemia en nuestro grupo de pacientes cuando aparecía alguna de las siguientes alteraciones: niveles de colesterol total superior a $200 \mathrm{mg} / \mathrm{dl}$, niveles de triglicéridos que excedían los $150 \mathrm{mg} / \mathrm{dl}$, HDL colesterol inferior a 35 $\mathrm{mg} / \mathrm{dl}$ en el varón o $40 \mathrm{mg} / \mathrm{dl}$ en la mujer y LDL colesterol superior a $130 \mathrm{mg} / \mathrm{dl}$, en 2 determinaciones distintas.

Se recogieron los tratamientos relacionados con los factores de riesgo cardiovascular asociados.

\section{ANALISIS ESTADÍSTICO}

Para el análisis estadístico se realizó en primer lugar una descripción univariada de los datos para comprobar la calidad de los mismos, detectar errores de codificación y plantear posibilidades de categorización de variables cuantitativas o agrupación de categorías en las cualitativas. Para el análisis bivariante de dos variables cualitativas, se empleó el análisis de tablas de contingencia. El análisis se realizó mediante la prueba de Chi cuadrado de Pearson; en el caso de variables dicotómicas, se calculó también la odds ratio a un nivel de confianza del $95 \%$. En las variables cuantitativas se realizó un estudio estadístico descriptivo con la determinación de la media aritmética y la desviación típica. Se comprobó el ajuste a una distribución normal mediante prueba de KolmogorovSmirnov.

En el caso de una variable cuantitativa y otra cualitativa dicotómica, se utilizó el test t de Student para muestras independientes. En todos los casos se ha manejado un error alfa de 0,05 (considerándose estadísticamente significativo los valores de $\mathrm{p}<0,05)$.

Para el análisis multivariante se ha ajustado un modelo de regresión logística teniendo en cuenta las variables que fueron significativas $(\mathrm{p}<0,05)$ en el análisis bivariante. El método utilizado ha sido el de "Introducir" y se han ido seleccionando las variables en el modelo de acuerdo a su relación clínica. En el caso de variables categóricas se ha tomado el contraste "Indicador" que consiste en crear tantas variables dicotómicas (variables 0/1) como categorías menos 1, reciben el nombre de variables indicadoras y las categorías de la variable predictora se van comparando con la elegida, en nuestro caso la primera.

El programa utilizado para el análisis ha sido el SPSS 11.5 (Statical Package for Social Science) (SPSS, Inc., IL., USA).

\section{RESULTADOS}

\section{CARACTERÍSTICAS SOCIODEMOGRÁFICAS DE LA MUESTRA}

Se recogieron los datos sociodemográficos de la muestra $(\mathrm{n}=330)$ con una edad media de 74, $78 \pm 10,35$ años (el $51,8 \%$ mayores de 75 años), siendo la edad de las mujeres mayor $(78,88 \pm 10,45$ años) que la de los hombres $(73,70 \pm$ $10,07)(\mathrm{p}<0,0001)$, con una distribución por sexos, de 261 hombres $(79,1 \%)$ y 69 mujeres $(20,9 \%)$.

\section{PREVALENCIA DE FACTORES DE RIESGO CARDIOVASCULAR}

Se observó que la población tenía un índice de masa corporal (IMC) (Tabla I) medio de $25,42 \pm 4,08 \mathrm{~kg} / \mathrm{m} 2$. Comparamos los datos según el sexo, encontrando que éste era mayor en mujeres (IMC $=26,8 \pm 4,8$ ) que en hombres (IMC $=25,1 \pm 3,9)$ con una diferencia significativa $(\mathrm{p}$ $=0,014)$. Si analizamos el porcentaje de pacientes obesos $($ IMC $\geq 30)$ observamos una prevalencia del $12,2 \%$ de obesidad, donde el $20,9 \%$ de las mujeres y el $10,3 \%$ de los hombres tienen un IMC $\geq 30$, sin diferencias significativas. 
TABLA I

FACTORES DE RIESGO CARDIOVASCULAR

\begin{tabular}{|c|c|c|c|c|}
\hline & Total & Hombres & Mujeres & $p$ \\
\hline Parámetros & Media & $\pm D T$ & & \\
\hline $\begin{array}{l}\text { IMC (peso/talla²) } \\
\text { PAS (mmHg) } \\
\text { PAD (mmHg) } \\
\text { Colesterol }\end{array}$ & $\begin{array}{r}25,4 \pm 4,1 \\
136,9 \pm 31,5 \\
75,7 \pm 15,1\end{array}$ & $\begin{array}{c}25,1 \pm 3,9 \\
135,0 \pm 30,1 \\
75,0 \pm 14,7\end{array}$ & $\begin{array}{c}26,8 \pm 4,8 \\
144,0 \pm 35,7 \\
78,3 \pm 16,0\end{array}$ & $\begin{array}{l}0,014 \\
0,034 \\
\text { NS }\end{array}$ \\
\hline $\begin{array}{l}\text { Total (mg/dl) } \\
\text { Colesterol }\end{array}$ & $168,7 \pm 42,3$ & $165,7 \pm 41,4$ & $180,1 \pm 43,9$ & 0,017 \\
\hline $\begin{array}{l}\mathrm{HDL}(\mathrm{mg} / \mathrm{dl}) \\
\text { Colesterol }\end{array}$ & $40,0 \pm 44,7$ & $36,2 \pm 11,5$ & $55,0 \pm 96,3$ & NS \\
\hline LDL (mg/dl) & $113,2 \pm 34,7$ & $111,6 \pm 33,3$ & $119,5 \pm 39,5$ & NS \\
\hline $\begin{array}{l}\text { Triglicéridos } \\
(\mathrm{mg} / \mathrm{dl}) \\
\text { Clucosa }\end{array}$ & $120,4 \pm 56,2$ & $119,8 \pm 57,1$ & $125,4 \pm 53,1$ & NS \\
\hline $\begin{array}{l}\text { Plasmática } \\
(\mathrm{mg} / \mathrm{dl})\end{array}$ & $121,9 \pm 55,8$ & $117,8 \pm 52,9$ & $137,3 \pm 63,4$ & 0,010 \\
\hline
\end{tabular}

DT: desviación típica. IMC; índice de masa corporal; PAS: presión arterial sistólica; PAD: presión arterial diastólica.

La presión arterial sistólica media fue de 136,91 \pm 31,53 $\mathrm{mmHg}$, con diferencias significativas en relación al sexo del paciente, ya que la presión media en las mujeres fue mayor $(144,0 \pm 35,7 \mathrm{mmHg})$ que en los hombres $(135,0 \pm 30,1 \mathrm{mmHg})$ $(\mathrm{p}=0,034)$. En cuanto a la presión arterial diastólica media fue $75,7 \pm 15,1 \mathrm{mmHg}$, pero no encontramos diferencias significativas entre ambos sexos. El 37,4 \% de la población $(\mathrm{n}=123)$ presentaban hipertensión arterial. Al analizar la distribución por sexos según la prevalencia de hipertensión arterial se encontró que el $36,2 \%$ de los hombres eran hipertensos $(n=94)$ frente al $42,0 \%$ de las mujeres $(n=29)$, sin ser significativas estas diferencias. De los 123 pacientes hipertensos de la muestra, solo 78 $(63,4 \%)$ estaban tratados farmacológicamente. Recogimos los fármacos empleados en aquellos hipertensos que recibían tratamiento para controlar las cifras de presión arterial, un 3\% recibían diuréticos, un 26,9\% IECAS, un 19,2\% calcioantagonistas y un 20,5\% terapia combinada con 2 o más fármacos.

En relación al perfil lipídico, las cifras medias de colesterol total se encontraban dentro del rango de la normalidad, $168,72 \pm 42,26 \mathrm{mg} / \mathrm{dl}$, así como los valores medios de las subfracciones colesterol HDL y colesterol LDL (40,03 \pm 44,70 $\mathrm{mg} / \mathrm{dl}$ y $113,23 \pm 34,68 \mathrm{mg} / \mathrm{dl}$, respectivamente). Al comparar ambos sexos, sólo encontramos diferencias estadísticamente significativas en los valores medios de colesterol total, que eran más elevados en la mujeres $(180,1 \pm 43,9 \mathrm{mg} / \mathrm{dl})$ con respecto a los hombres $(165,7 \pm 41,4 \mathrm{mg} / \mathrm{dl})(\mathrm{p}=0,017)$. Recogimos los datos relacionados con el metabolismo lipídico y encontramos que el 59,9\% ( $\mathrm{n}=178)$ de la población del estudio presentaba dislipemia frente al 40,1\% $(\mathrm{n}=119)$ que tenía valores de triglicéridos, colesterol total y subfracciones de colesterol dentro de la normalidad. Los pacientes con dislipemia se distribuyeron según el sexo de la siguiente forma: el $61,7 \%$ de los varones eran dislipémicos $(n=145)$ frente al $53,2 \%$ de las mujeres $(n=33)$, sin diferencias significativas. Un dato relevante es que de los 178 pacientes con dislipemia, solo el $4,5 \%$ estaban tratados (4,2\% estatinas y $0,3 \%$ fibratos).
Los niveles medios de glucosa plasmática fueron de 121,9 $\pm 55,8 \mathrm{mg} / \mathrm{dl}$, siendo más altos en las mujeres $(137,3 \pm 63,4$ $\mathrm{mg} / \mathrm{dl})$ con respecto a los hombres $(117,8 \pm 52,9 \mathrm{mg} / \mathrm{dl})$, de forma estadísticamente significativa $(\mathrm{p}=0,010)$. El 42,4\% (n $=140$ ) de la población presentaba diabetes mellitus y todos eran diabéticos tipo 2, frente al $57,6 \%(\mathrm{n}=190)$ que no padecían esta enfermedad. Al estudiar la distribución por sexos en los pacientes diabéticos encontramos un mayor porcentaje de mujeres con diabetes mellitus tipo $2(56,5 \%)$ frente al menor porcentaje de hombres diabéticos $(38,7 \%)$, siendo estas diferencias estadísticamente significativas $(p=0,009)$. De los 140 pacientes diabéticos, el $30,7 \%$ presentaba otras complicaciones de la diabetes mellitus, además de la arteriopatía crónica periférica, las cuales se muestran en la tabla II. En cuanto al tratamiento de la diabetes, más de la mitad de la muestra recibía tratamiento insulínico $(64,3 \%)$, un 30\% recibían hipoglucemiantes orales y el 5,7\% en el momento del ingreso recibía únicamente tratamiento dietético.

\section{TABLA II}

OTRAS COMPLICACIONES DE LA DIABETES MELLITUS TIPO 2

\begin{tabular}{lccc}
\hline Parámetros & $\begin{array}{c}\text { Total } \\
\text { (n) } \%\end{array}$ & $\begin{array}{c}\text { Hombres } \\
\text { (n) } \%\end{array}$ & $\begin{array}{c}\text { Mujeres } \\
\text { (n) } \%\end{array}$ \\
\hline Retinopatía & $(15) 10,7 \%$ & $(9) 6,4 \%$ & (6) $4,2 \%$ \\
Nefropatía & $(12) 8,6 \%$ & $(9) 6,4 \%$ & $(3) 2,1 \%$ \\
Coronariopatía & $(9) 6,4 \%$ & $(7) 5,0 \%$ & $(2) 1,4 \%$ \\
ACV & $(7) 5,0 \%$ & $(6) 4,3 \%$ & $(1) 0,7 \%$ \\
Polineuropatía & $(99) 70,7 \%$ & $(67) 66,3 \%$ & $(32) 82,1 \%$ \\
\hline
\end{tabular}

ACV: Accidente cerebrovascular. No existen diferencias estadísticamente significativas.

Con respecto a los hábitos tóxicos más de la mitad de la muestra, el 52,1\%, no fumaba, sólo el 19,1\% eran fumadores y el 28,8\% habían abandonado el hábito de fumar. Al comparar el hábito tabáquico según el sexo, encontramos diferencias significativas entre los hombres y las mujeres $(p<0,0001)$. Fumaban en un mayor porcentaje los hombres $(23,0 \%)$ que las mujeres $(4,3 \%)$. En cuanto al consumo de cigarrillos, se observó que el 3,9\% de la muestra fumaba menos de 10 cigarrillos/día, el 8,2\% fumaba entre 11 y 20 cigarrillos/día y el $7,0 \%$ fumaba más de 20 cigarrillos/día.

Un $85,8 \%$ de la población refería no beber alcohol de forma habitual, el 5,2\% había abandonado el consumo y el 9,1\% de la población lo consumía de forma habitual. Al relacionar el hábito enólico con el sexo, encontramos diferencias significativas en cuanto al consumo habitual de alcohol $(p=0,006)$, una mayor proporción de hombres $(11,5 \%)$ consumían alcohol de forma habitual en relación a las mujeres, que contestaron todas negativamente.

El $35,2 \%$ de los pacientes de nuestro estudio presentaban dolor en reposo en el momento del ingreso, lo cual corresponde a pacientes en estadio clínico III, de ellos el 40,6\% eran hombres frente al 14,5\% que eran mujeres. Sin embargo, más de la mitad de la muestra, el 64,8\%, presentaba lesiones tróficas y/o gangrena, correspondiéndose con el estadio IV de Fontaine $(59,4 \%$ de hombres vs. $85,5 \%$ mujeres). La media del ITB fue en el grupo global de $0,3 \pm 0,2$, en los varones de 
TABLA III

MODELO DE REGRESIÓN LOGÍSTICA PARA EL ESTADIO CLÍNICO

\begin{tabular}{lccccc}
\hline & & & & \multicolumn{2}{c}{ IC 95\% para exp $(B)$} \\
\cline { 5 - 6 } Variable & $B$ & Sig. & $\operatorname{Exp}(B)$ & Inferior & Superior \\
\hline Diabetes mellitus & 1,201 & 0,000 & 3,322 & 1,881 & 5,866 \\
Colesterol total & $-0,010$ & 0,000 & 0,990 & 0,986 & 0,994 \\
$\begin{array}{l}\text { Sitio de la lesión } \\
\quad \text { Proximal }\end{array}$ & & & & & \\
$\quad$ Distal & 1,931 & 0,000 & & & \\
$\quad$ Ambas & 1,264 & 0,000 & 6,897 & 3,509 & 13,557 \\
Sexo & 1,077 & 0,009 & 2,538 & 1,346 & 9,297 \\
Edad $>75$ años & 0,463 & 0,094 & 1,589 & 1,302 & 6,623 \\
\hline
\end{tabular}

$0,28 \pm 0,20$ y en las mujeres de $0,26 \pm 21$, sin encontrar diferencis significativas entre los sexos.

Los pacientes con isquemia crónica de miembros inferiores fueron intervenidos quirúrgicamente, en un intento de revascularizar los tejidos isquémicos, una media de $2,2 \pm 1,7$ veces. $\mathrm{El}$ $81,8 \%$ de los pacientes en estadio clínico IV ha sufrido una amputación en el miembro inferior mientras que sólo el 6,9\% de los que han sufrido este tipo de intervención quirúrgica se encontraban en estadio III. Por el contrario, el 93,1\% de los pacientes en estadio clínico III han sido intervenidos con técnicas de revascularización frente al 18,2\% en estadio clínico IV, siendo estas diferencias estadísticamente significativas.

El modelo de regresión logística construido para explicar el estadio clínico dicotomizado en III/IV se presenta en la tabla III. En el modelo de regresión logística, ajustado para la edad y sexo, las variables que mayor influencia tienen en el riesgo de padecer un estadio IV frente al III son la diabetes mellitus, la localización de la lesión arterial, y las cifras bajas de colesterol total.

Un paciente con diabetes mellitus tipo 2 tiene 3,322 (IC 95\%: 1,881-5,866) veces más riesgo de presentar al ingreso un estadio clínico IV que una persona que no lo es.
En cuanto al colesterol total, la OR es de 0,990 lo cual puede interpretarse como que el riesgo de presentar un estadio clínico IV frente al estadio clínico III se multiplicaría por 0,990 por cada $\mathrm{mg} / \mathrm{dl}$ de aumento del nivel de colesterol total. Es decir, si hallamos el inverso, la probabilidad de presentar un estadio clínico IV por cada $\mathrm{mg} / \mathrm{dl}$ de disminución de colesterol total es de 1,01 (IC 95\%: 1,014-1,006). La localización de la lesión arterial distal (por debajo del ligamento inguinal) incrementa el riesgo de presentar un estadio clínico IV en 6,897 (IC 95\%: 3,509-13,557) veces más que la localización de la lesión arterial en otro sector.

El modelo de regresión logística construido para explicar el tipo de cirugía dicotomizado en amputaciones/derivaciones se presenta en la tabla IV. En el modelo de regresión logística, ajustado para la edad y sexo, la presencia de diabetes mellitus tipo 2 presenta una odds ratio (OR) de 3,373 lo que significa que el padecimiento de esta enfermedad multiplica por 3,37 (IC 95\%: 1,940-5,866) el riesgo de ser sometido a una amputación frente a una revascularización. Con respecto del colesterol total, la OR es de 0,986 lo cual puede interpretarse como que el riesgo de amputación frente a intervenciones de revascularización se multiplicaría por 0,986 por cada $\mathrm{mg} / \mathrm{dl} \mathrm{de}$

MODELO DE REGRESIÓN LOGÍSTICA PARA EL TIPO DE CIRUGÍA

\begin{tabular}{lccccc}
\hline & & & & \multicolumn{2}{c}{$I C$ 95\% para exp $(B)$} \\
\cline { 5 - 6 } Variable & $B$ & Sig. & Exp $(B)$ & Inferior & Superior \\
\hline Diabetes mellitus & 1,216 & 000 & 3,373 & 1,940 & 5,866 \\
Colesterol total & $-0,014$ & 0,000 & 0,986 & 0,982 & 0,990 \\
Sitio lesión & & & & & \\
$\quad$ Proximal & 0,950 & 0,012 & & & \\
$\quad$ Distal & 0,005 & 2,585 & 1,324 & 3,047 \\
$\quad$ Ambas & 1,103 & 0,631 & 1,282 & 0,474 & 5,666 \\
Más de una cirugía & 0,008 & 0,032 & 1,008 & 1,602 & 1,016 \\
Días ingreso & 0,522 & 0,058 & 1,686 & 0,001 & 2,891 \\
Edad >75 años & 0,418 & 0,236 & 1,520 & 0,761 & 3,034 \\
Sexo & & & & & \\
\hline
\end{tabular}


aumento del nivel de colesterol total. Dicho de otra forma el riesgo de amputación se incrementaría en un 1,4\% (1/0,986 = $1,014)$ por cada $\mathrm{mg} / \mathrm{dl}$ de disminución en el nivel de colesterol. En cuanto al sitio de la lesión aunque la variable entra globalmente en el modelo, las diferencias significativas se sitúan en la comparación entre lesión distal y proximal siendo la OR de la primera de 2,585 en relación a la segunda. Es decir que los individuos con una lesión distal (por debajo del ligamento inguinal) multiplican por 2,585 el riesgo de sufrir una amputación frente a aquellos que su lesión es de localización proximal (sector aorto-ilíaco).

Las personas que han sufrido más de una intervención quirúrgica presentan una $\mathrm{OR}$ de $3,013(1,602-5,666)$ para sufrir una amputación frente a aquellas que han sido intervenidas una sola vez. Cada día de ingreso hospitalario aumenta en un 0,8\% el riesgo de amputación frente a intervenciones de revascularización.

\section{DISCUSIÓN}

A pesar de su elevada prevalencia los estudios que analizen la relación entre los diferentes factores clinico bioquimicos y el esatdiaje clinico así como el tipo de cirugía realizada en esta población son escasos.

Según nuestros resultados, las variables que tienen mayor influencia, en el modelo de regresión logística, ajustado para la edad y sexo, para presentar un estadio clínico IV de arteriopatía crónica periférica frente al estadio clínico III son la diabetes mellitus, la localización de la lesión arterial y cifras bajas de colesterol sérico total.

Numerosos estudio muestran que los pacientes con diabetes mellitus tienen más probabilidad de presentar úlceras o gangrenas que los pacientes no diabéticos. En el estudio de Dormandy y cols. (10), encontraron un peor pronóstico en los pacientes diabéticos con lesiones tróficas (estadio clínico IV de arteriopatía periférica), ya que el riesgo de amputación en estos pacientes era 10 veces superior y a una edad más temprana que en los pacientes no diabéticos. En el primer estudio prospectivo español, que analiza los factores de riesgo cardiovascular en la enfermedad arterial periférica (11), la diabetes mellitus en el análisis de regresión logística representa un riesgo de 5,1 para desarrollar enfermedad arterial periférica en los varones y de 5,9 en las mujeres. Estos datos han sido confirmados en otros trabajos (12). Si comparamos estos resultados con los de nuestro estudio, encontramos que en nuestro trabajo el riesgo de tener isquemia crítica de miembros inferiores si se es diabético es algo inferior (OR de 3,322) que el encontrado en el estudio prospectivo, quizá debido a que en éste último incluyen pacientes no solo con isquemia crítica de miembros inferiores sino también pacientes con grados menos avanzados de arteriopatía crónica periférica.

En nuestro estudio, llama la atención que los pacientes con un estadio clínico IV o más avanzado, tienen cifras medias más bajas de colesterol sérico total que los pacientes con un estadio clínico III. Este fenómeno se ha descrito en el estudio de Bismuth y cols. (13) el cual encontró cifras bajas de colesterol total en pacientes con isquemia crítica de miembros inferiores. En el estudio español de Marín y cols. (11) también encontraron cifras menores de colesterol total en los pacientes con isquemia crítica de miembros inferiores (grado III y IV de Leriche y Fontaine) con respecto a los pacientes con claudicación intermitente.
La localización de la lesión arterial tiene cierto valor predictivo. Esto se pone de manifiesto al revisar los resultados de otros autores, como por ejemplo el estudio del grupo italiano de estudio de la isquemia crítica (14), encontraron que las lesiones infrainguinales (lesiones femoro-poplíteas) se asociaron con una menor mortalidad que las lesiones distales (distal a la poplítea) [un riesgo relativo de mortalidad a los 12 meses de las lesiones de localización infrainguinal de 0,74 con respecto a las distales]; sin embargo las lesiones distales fueron predictivas de mayor proporción de amputaciones con respecto a las lesiones infrainguinales RR 0,66. En nuestro estudio, demostramos el mayor riesgo de estadio IV en las lesiones infrainguinales

En el estudio multivariante, las variables que se relacionan de forma significativa para relacionar el tipo de intervención quirúrgica dicotomizada en amputaciones y revascularizaciones, fueron la diabetes mellitus tipo 2, el colesterol total, la localización de la obstrucción (diferencias significativas al comparar localización proximal y distal), el número de intervenciones quirúrgicas y los días de ingreso hospitalario.

En nuestro estudio, los pacientes con diabetes mellitus tipo 2 tienen 3,373 veces más riesgo de sufrir una amputación que los pacientes no diabéticos. Estos datos han sido confirmados en otros trabajos (21), con valores más elevados OR 22 comparados con los no diabéticos. Si bien es cierto, que este estudio estima la incidencia de amputaciones en diabéticos comparados con los no diabéticos y no la prevalencia, como ocurre nuestro estudio. Los mismos autores antes analizados, publican un estudio posterior, de casos controles (22) para comparar el riesgo de amputaciones en los pacientes diabéticos comparados con la población general, y cuyos resultados del análisis de regresión logística mostraron que, ajustado para la edad y sexo, el riesgo de amputación en los pacientes diabéticos fue de 18,2 (OR 18,2) comparados con la población general.

Según datos del Medicare, en Estado Unidos, el riesgo relativo de sufrir una amputación en los pacientes diabéticos es del 12,7 y se duplica entre los 65 y los 74 años (17), estos datos han sido confrimados en otras areas geográficas $(18,19)$.

Uno de los resultados más llamativos de nuestro trabajo fue encontrar que las cifras de colesterol total en los pacientes con un grado más avanzado de arteriopatía periférica y amputaciones, eran más bajos que los pacientes con menor grado de arteriopatía periférica e intervenciones de revascularización. El análisis de regresión logística mostró una OR de 0,986 para la relación entre las cifras bajas de colesterol total y el riesgo de amputación. Estos hallazgos son similares a los resultados del estudio dirigido por Jean Bismuth et al (13), en el cual los niveles de lípidos séricos se encuentran disminuidos en los pacientes con isquemia crítica de miembros inferiores, en el período perioperatorio (antes de la intervención quirúrgica y 3 meses después de ésta), relacionándose inversamente con los reactantes de la fase aguda (albúmina, proteína C-reactiva). Confirmandose estos datos en el estudio prospectivo nacional (11). Según los resultados de estos estudios y los nuestros podemos concluir que el papel del colesterol total en la isquemia crítica de miembros inferiores es incierto, quizá porque ha sido escasamente investigado. Con estos resultados solo podemos interpretar que existe una asociación estadística entre los niveles más bajos de colesterol total en los pacientes con graves lesiones arteriales o con amputaciones en miembros inferiores, lo cual puede ser una referencia para plantear nuevas hipótesis de estudio. 
La ubicación de la obstrucción en el árbol arterial tiene cierto valor predictivo positivo. Las obstrucciones de troncos distales se asocian con una tasa más alta de amputación y de persistencia de la isquemia crítica. En nuestro estudio encontramos que los individuos con una lesión distal, por debajo del ligamento inguinal, tienen un riesgo de 2,585 de sufrir una amputación del miembro inferior que los pacientes con lesión arterial proximal. Estos resultados son similares a los obtenidos por un grupo del Reino Unido (19) que encontraron que las amputaciones menores y mayores eran más frecuentes en aquellos pacientes con lesiones por debajo del ligamento inguinal (RR 3,32 y RR 4,24, respectivamente).

Los resultados del análisis multivariante muestran que los pacientes que han sufrido más de una intervención quirúrgica presentan 3,013 veces más riesgo de sufrir una amputación en el miembro inferior que aquellos que han sido intervenidos en una ocasión. Esta exceso de riesgo se debe probablemente, a los numerosos intentos de revascularizar antes de proceder a una amputación. Otras de las variables que se incluyen en el modelo de regresión logística son los días de ingreso hospitalario. Por cada días de ingreso aumenta un $0,8 \%$ el riesgo de amputación frente a intervenciones de revascularización. Esto podría explicarse ya que las estancias de estos pacientes en el hospital son elevadas y ello conlleva a un mayor número de complicaciones como infecciones hospitalarias, entre otras. Teniendo en cuenta la alta prevalencia de diabetes mellitus tipo 2, las complicaciones en estos pacientes pueden ser más frecuentes y de mayor duración y control.

En conclusión, en el análisis multivariante la presencia de diabetes mellitus, lesiones distales, sexo masculino y edad mayor de 75 se relaciona con la presencia de un estadio IV, presentando los niveles de colesterol una relación inversa. La presencia de diabetes mellitus, lesion distal, más de una cirugía reparadora, edad mayor de 75 años, los dias de ingreso se relacionan con al realización de amputaciones como técnica reparadora, presentando los niveles de colesterol una relación inversa.

\section{Bibliografía}

1. TASC (TransAtlantic Inter.-Society) working group, Consensus. Management of peripheral arterial disease (PAD). J Vasc Surg 2000; 31 (S1): 1-296.

2. Pedrini L.Critical ischaemia of the lower limbs: diagnosis and therapeutic strategies. Foot and Ankle Surgery 2003; 9: 87-94.

3. Dormandy J, Heek L, Vig S. Predicting which patients will develop chronic critical leg ischaemia. Sem Vasc Surg 1999; 12: 138-141.

4. Planas A, Clará A, Marrugat J, Pou JM, Gasol A, de Moner A, et al. Age at onset of smoking is an independent risk factor in peripheral artery disease development. J Vasc Surg 2002; 35: 506-509.

5. Hiatt WR, Hoag S, Hammen RF. Effect of diagnostic criteria on the prevalence of peripheral arterial disease. Circulation 1995; 91: 1472-1479.

6. Calle Pascual AL, Duran A, Diaz A, Monux G, Serrano FJ, de la Torre NG, Moraga I. Comparison of peripheral arterial reconstruction in diabetic and non-diabetic patients: a prospective clinic-based study. Diabetes Res Clin Pract 2001;53:129-136.

7. Gruppo di studio dellíschemica cronica critica degli arti inferior. A prospective epidemiological survey of the natural history if chronic critical leg ischemia. The ICAI Group. Eur J Vasc Endovasc Surg 1996;11:112-120.

8. Gomes AS. Principles of angiography and interventional radiology. En: Moore WS, editor. Vascular surgery: a comprehensive review. Filadelfia: WB Saunders 1998; 266-303.

9. Fontaine R, Kim M, Kieny R. Die chirurgische Behandlung der peripheren Durch-blutungsstörungen. Helvetia Chirurgica Acta 1954; 5/6: 199-533.

10. Dormandy JA, Heeck L, Vig S. Predictors of early disease in the lower limbs. Semin Vasc Surg 1999; 12: 109-117.

11. Marín R, Fernández-Vega F, Escalada P, Estevan JM, Barreiro A, Álva- rez J. Factores de riesgo cardiovascular en la enfermedad arterial periférica. Estudio de 403 casos. Rev Clin Esp 1993; 193: 357-362.

12. Cheng SW, Ting AC, Lau H, Wong J. Survival in patiens with chronic lower extremity ischemia: A risk factor analysis. Ann Vasc Surg 2000; 14: $158-165$.

13. Bismuth J, Kofoed SC, Jensen AS, Sethi A, Sillesen H. Serum lipids act as inverse acute phase resctants and are falsely low in patient with critical limb ischemia. J Vasc Surg 2002; 36: 1005-1010.

14. Bertele V, Roncaglioni MC, Pangrazzi J, Tercian E, Tognoni EG. Clinical outcome and its predictors in 1560 patients with critical leg ischaemia. Chronic Critical Leg Ischaemia Group. Eur J Vasc Endovasc Surg 1999; 12: 138-141).

15. Trautner C, Haastert B, Spraul M, Giani G, Berger M. Unchanged incidence of lower-limb amputations in a Germany city. Diabetes Care 2001; 24: 855-859.

16. Trautner C, Haastert B, Giani G, Berger M. Amputations and diabetes: a case-control Study. Diab Med 2002; 19: 35-40.

17. Beckman JA, Creager MA, Libby P. Diabetes and atherosclerosis: epidemiology, pathophysiology and management. JAMA 2002; 287: 25702581.

18. Jude EB, Oyibo SO, Chalmers N, Boulton AJM. Peripheral arterial disease in diabetic and non-diabetic patients. A comparison of severity and outcome. Diabetes Care 2001; 24:1433-1437.

19. Gutteridge W, Torrie EP, Galland RB. Cumulative risk of bypass, amputation or death following percutaneous transluminal angioplasty. Eur J Vasc Endovasc Surg 1997; 13: 14-22.

20. Brugada R, Wenger NK, Jacobson TA, Clark WS, Cotsonis G, Iglesias A. Changes in plasma cholesterol levels after hospitalisation for acute coronary events. Cardiology 1996; 87: 194-199. 